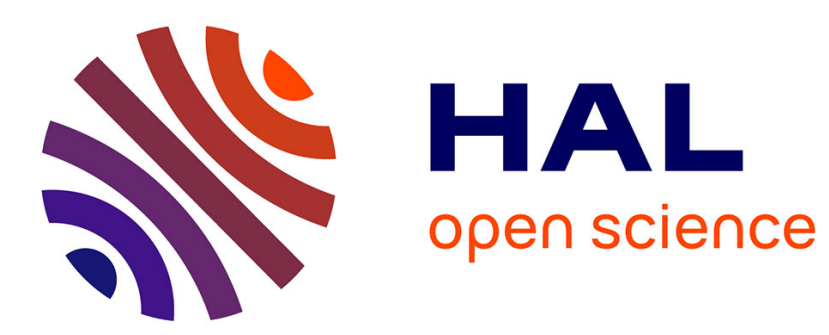

\title{
Multi-level fast multipole BEM for 3-D elastodynamics
}

Marc Bonnet, Stéphanie Chaillat, Jean-François Semblat

\section{To cite this version:}

Marc Bonnet, Stéphanie Chaillat, Jean-François Semblat. Multi-level fast multipole BEM for 3-D elastodynamics. Recent Advances in BEM, Springer, pp.15-28, 2009. hal-00326481v2

\section{HAL Id: hal-00326481 \\ https://hal.science/hal-00326481v2}

Submitted on 3 Oct 2008

HAL is a multi-disciplinary open access archive for the deposit and dissemination of scientific research documents, whether they are published or not. The documents may come from teaching and research institutions in France or abroad, or from public or private research centers.
L'archive ouverte pluridisciplinaire HAL, est destinée au dépôt et à la diffusion de documents scientifiques de niveau recherche, publiés ou non, émanant des établissements d'enseignement et de recherche français ou étrangers, des laboratoires publics ou privés. 


\title{
Multi-level fast multipole BEM for 3-D elastodynamics
}

\author{
Marc Bonnet and Stéphanie Chaillat and Jean-François Semblat
}

\begin{abstract}
To reduce computational complexity and memory requirement for 3-D elastodynamics using the boundary element method (BEM), a multi-level fast multipole BEM (FM-BEM) based on the diagonal form for the expansion of the elastodynamic fundamental solution is proposed and demonstrated on numerical examples involving single-region and multi-region configurations where the scattering of seismic waves by a topographical irregularity or a sediment-filled basin is examined.
\end{abstract}

\section{Introduction}

The boundary element method (BEM) is a mesh reduction method, subject to restrictive constitutive assumptions but yielding highly accurate solutions. It is in particular well suited to unbounded-domain idealizations commonly used e.g. in seismic wave modelling $[6,13]$. Many early references on BEMs and their application to elastodynamics can be found in the review articles $[1,2]$ and in e.g. [3].

To reduce computational complexity and memory requirement for 3-D elastodynamics using the boundary element method (BEM), a multi-level fast multipole BEM (FM-BEM) treatment is proposed (see e.g. $[12,16]$ for expositions of FMBEM for Helmholtz-type problems and related references). By adapting to this context recent implementations of the FMM for the Maxwell equations [7, 21], it brings significant improvement over previously published elastodynamics FM-BEM [11]. The diagonal form [18] for the expansion of the elastodynamic fundamental solution is used, with a truncation parameter adjusted to the subdivision level, a feature necessary for achieving optimal computational efficiency. The formulation is extended to problems featuring piecewise-homogeneous media via a multi-region FM-BEM

Marc Bonnet, Stéphanie Chaillat

LMS, Ecole Polytechnique, France, e-mail: [bonnet,chaillat]@1ms.polytechnique.fr

Jean-François Semblat

LCPC, Paris, France e-mail: semblat@lcpc.fr 
whose unknowns feature displacements and tractions on interfacial boundary elements. The correctness and computational performances of the proposed singleand multi-region versions of the elastodynamic FMM are demonstrated here on numerical examples featuring up to $O\left(2 \times 10^{5}\right)$ DOFs run on a single-processor PC, including a 3-D site effect benchmark (semi-spherical empty canyon or sedimentfilled basin, with previously published results $[15,17,20]$ for low-frequency cases allowing comparisons).

\section{Elastodynamic boundary element method}

Let $\Omega \subset \mathbb{R}^{3}$ denote the region of space occupied by a three-dimensional elastic solid with isotropic constitutive properties defined by $\mu$ (shear modulus), $\nu$ (Poisson's ratio) and $\rho$ (mass density). Time-harmonic motions, with circular frequency $\omega$, induced by a prescribed traction distribution $\boldsymbol{t}^{D}$ on the boundary $\partial \Omega$ and in the absence of body forces are considered for ease of exposition, other boundary conditions needing only simple modifications to the treatment presented therein. The displacement $\boldsymbol{u}$ on the boundary is governed by the well-known integral equation:

$$
(\mathcal{K} u)(\boldsymbol{x})=f(\boldsymbol{x}) \quad(\boldsymbol{x} \in \partial \Omega),
$$

with the linear integral operator $\mathcal{K}$ and the right-hand side $f$ defined by

$$
\begin{aligned}
(\mathcal{K} u)(\boldsymbol{x}) & =c_{i k}(\boldsymbol{x}) u_{i}(\boldsymbol{x})+(\text { P.V. }) \int_{\partial \Omega} u_{i}(\boldsymbol{y}) T_{i}^{k}(\boldsymbol{x}, \boldsymbol{y}) d S_{y} \\
f(\boldsymbol{x}) & =\int_{\partial \Omega} t_{i}^{D}(\boldsymbol{y}) U_{i}^{k}(\boldsymbol{x}, \boldsymbol{y}) d S_{y},
\end{aligned}
$$

where (P.V.) indicates a Cauchy principal value (CPV) singular integral and the freeterm $c_{i k}(\boldsymbol{x})$ is equal to $0.5 \delta_{i k}$ in the usual case where $\partial \Omega$ is smooth at $\boldsymbol{x}$. Moreover, $U_{i}^{k}(\boldsymbol{x}, \boldsymbol{y})$ and $T_{i}^{k}(\boldsymbol{x}, \boldsymbol{y})$ denote the $i$-th components of the elastodynamic fundamental solution [9], i.e. of the displacement and traction, respectively, generated at $\boldsymbol{y} \in \mathbb{R}^{3}$ by a unit point force applied at $\boldsymbol{x} \in \mathbb{R}^{3}$ along the direction $k$ :

$$
\begin{aligned}
U_{i}^{k}(\boldsymbol{x}, \boldsymbol{y}) & =\frac{1}{k_{\mathrm{S}}^{2} \mu}\left(\left(\delta_{q s} \delta_{i k}-\delta_{q k} \delta_{i s}\right) \frac{\partial}{\partial x_{q}} \frac{\partial}{\partial y_{s}} G\left(r ; k_{\mathrm{S}}\right)+\frac{\partial}{\partial x_{i}} \frac{\partial}{\partial y_{k}} G\left(r ; k_{\mathrm{P}}\right)\right), \\
T_{i}^{k}(\boldsymbol{x}, \boldsymbol{y}) & =\mu\left[\frac{2 \nu}{1-2 \nu} \delta_{i j} \delta_{h \ell}+\delta_{i h} \delta_{j \ell}+\delta_{j h} \delta_{i \ell}\right] \frac{\partial}{\partial y_{\ell}} U_{h}^{k}(\boldsymbol{x}, \boldsymbol{y}) n_{j}(\boldsymbol{y})
\end{aligned}
$$

in which $r=|\boldsymbol{y}=\boldsymbol{x}|$ and $k_{\mathrm{S}}, k_{\mathrm{P}}$ are the respective $\mathrm{S}$ and $\mathrm{P}$ wavenumbers, so that

$$
k_{\mathrm{S}}^{2}=\frac{\rho \omega^{2}}{\mu}, \quad k_{\mathrm{P}}=\gamma k_{\mathrm{S}}, \quad \gamma^{2}=\frac{1-2 \nu}{2(1-\nu)},
$$


$G(\cdot ; k)$ is the free-space Green's function for the Helmholtz equation with wavenumber $k$, given by

$$
G(r ; k)=\frac{\exp (\mathrm{i} k r)}{4 \pi r},
$$

$\boldsymbol{n}(\boldsymbol{y})$ is the unit normal to $\partial \Omega$ directed outwards of $\Omega$.

The numerical solution of boundary integral equation (1) is based on a discretization of the surface $\partial \Omega$ into isoparametric boundary elements, with piecewise-linear interpolation of displacements and piecewise-constant interpolation of tractions, based on three-noded triangular boundary elements, being used here. On collocating (1) at displacement nodes, a square complex-valued matrix equation of the form

$$
[K]\{u\}=\{f\},
$$

is obtained, where the $N$-vector $\{u\}$ collects the sought degrees of freedom (DOFs), here the nodal displacement components, while the $N \times N$ matrix of influence coefficients $[K]$ and the $N$-vector $\{f\}$ arise from (2) and (3), respectively. Setting up the matrix $[K]$ classically requires the computation of all element integrals for each collocation point, thus needing a computational time of order $O\left(N^{2}\right)$.

The influence matrix $[K]$ is fully-populated. Storing $[K]$ is thus limited, on ordinary computers, to BEM models of size not exceeding $N=O\left(10^{4}\right)$. Direct solvers, such as the LU factorization, have a $O\left(N^{3}\right)$ complexity and are thus also limited to moderately-sized BEM models. Both limitations are overcome by (i) resorting to an iterative solver, here GMRES [19], and (ii) accelerating the matrix-vector products $[K]\{u\}$ requested by each iteration of GMRES using the fast multipole method (FMM) so as the complexity of this operation becomes lower than the $O\left(N^{2}\right)$ operations entailed by standard BEM methods.

\section{Elastodynamic Fast Multipole Method}

The FMM is based on a reformulation of the fundamental solutions in terms of products of functions of $\boldsymbol{x}$ and of $\boldsymbol{y}$. This allows to re-use integrations with respect to $\boldsymbol{y}$ when the collocation point $\boldsymbol{x}$ is changed, thereby lowering the $O\left(N^{2}\right)$ complexity per iteration entailed by standard BEMs. The elastodynamic fundamental solutions $(4 \mathrm{a}, \mathrm{b})$ are linear combinations of derivatives of the Green's function (6) for the Helmholtz equation. On recasting the position vector $\boldsymbol{r}=\boldsymbol{y}-\boldsymbol{x}$ in the form $\boldsymbol{r}=\boldsymbol{r}_{0}+\left(\boldsymbol{y}-\boldsymbol{y}_{0}\right)-\left(\boldsymbol{x}-\boldsymbol{x}_{0}\right)$, where $\boldsymbol{x}_{0}$ and $\boldsymbol{y}_{0}$ are two poles and $\boldsymbol{r}_{0}=\boldsymbol{y}_{0}-\boldsymbol{x}_{0}$ (Fig. 1), the Helmholtz Green's function is shown [8] to admit the decomposition

$$
G(|\boldsymbol{r}| ; k)=\lim _{L \rightarrow+\infty} \int_{\hat{\boldsymbol{s}} \in S} e^{\mathrm{i} k \hat{\boldsymbol{s}} \cdot\left(\boldsymbol{y}-\boldsymbol{y}_{0}\right)} \mathcal{G}_{L}\left(\hat{\boldsymbol{s}} ; \boldsymbol{r}_{0} ; k\right) e^{-\mathrm{i} k \hat{\boldsymbol{s}} .\left(\boldsymbol{x}-\boldsymbol{x}_{0}\right)} \mathrm{d} \hat{\boldsymbol{s}},
$$

where $S$ is the unit sphere of $\mathbb{R}^{3}$ and the transfer function $\mathcal{G}_{L}\left(\hat{\boldsymbol{s}} ; \boldsymbol{r}_{0} ; k\right)$ is defined in terms of the Legendre polynomials $P_{p}$ and the spherical Hankel functions of the 


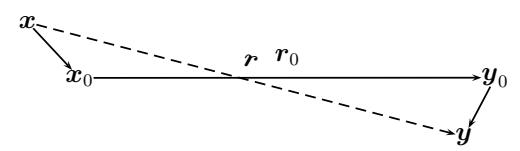

Fig. 1 Decomposition of the position vector: notation.

first kind $h_{p}^{(1)}$ by:

$$
\mathcal{G}_{L}\left(\hat{\boldsymbol{s}} ; \boldsymbol{r}_{0} ; k\right)=\frac{\mathrm{i} k}{16 \pi^{2}} \sum_{0 \leq p \leq L}(2 p+1) \mathrm{i}^{p} h_{p}^{(1)}\left(k\left|\boldsymbol{r}_{0}\right|\right) P_{p}\left(\cos \left(\hat{\boldsymbol{s}}, \boldsymbol{r}_{0}\right)\right) .
$$

The decomposition (8)-(9) is seen to achieve the desired separation of variables $\boldsymbol{x}$ and $\boldsymbol{y}$. Then, to recast the elastodynamic fundamental solutions in a form similar to (8)-(9), one simply substitutes decomposition (8)-(9) into $(4 \mathrm{a}, \mathrm{b})$, to obtain the following multipole decomposition of the elastodynamic fundamental solutions:

$$
\begin{aligned}
U_{i}^{k}(\boldsymbol{x}, \boldsymbol{y})= & \lim _{L \rightarrow+\infty} \int_{\hat{\boldsymbol{s}} \in S} e^{\mathrm{i} k_{\mathrm{P}} \hat{\boldsymbol{s}} \cdot\left(\boldsymbol{y}-\boldsymbol{y}_{0}\right)} \mathcal{U}_{i, L}^{k, \mathrm{P}}\left(\hat{\boldsymbol{s}} ; \boldsymbol{r}_{0}\right) e^{-\mathrm{i} k_{\mathrm{P}} \hat{\boldsymbol{s}} \cdot\left(\boldsymbol{x}-\boldsymbol{x}_{0}\right)} \mathrm{d} \hat{\boldsymbol{s}} \\
& +\lim _{L \rightarrow+\infty} \int_{\hat{\boldsymbol{s}} \in S} e^{\mathrm{i} k_{\mathrm{s}} \hat{\boldsymbol{s}} \cdot\left(\boldsymbol{y}-\boldsymbol{y}_{0}\right)} \mathcal{U}_{i, L}^{k, \mathrm{~S}}\left(\hat{\boldsymbol{s}} ; \boldsymbol{r}_{0}\right) e^{-\mathrm{i} k_{\mathrm{S}} \hat{\boldsymbol{s}} \cdot\left(\boldsymbol{x}-\boldsymbol{x}_{0}\right)} \mathrm{d} \hat{\boldsymbol{s}}, \\
T_{i}^{k}(\boldsymbol{x}, \boldsymbol{y})= & \lim _{L \rightarrow+\infty} \int_{\hat{\boldsymbol{s}} \in S} e^{\mathrm{i} k_{\mathrm{P}} \hat{\boldsymbol{s}} \cdot\left(\boldsymbol{y}-\boldsymbol{y}_{0}\right)} \mathcal{T}_{i, L}^{k, \mathrm{P}}\left(\hat{\boldsymbol{s}} ; \boldsymbol{r}_{0}\right) e^{-\mathrm{i} k_{\mathrm{P}} \hat{\boldsymbol{s}} \cdot\left(\boldsymbol{x}-\boldsymbol{x}_{0}\right)} \mathrm{d} \hat{\boldsymbol{s}} \\
& +\lim _{L \rightarrow+\infty} \int_{\hat{\boldsymbol{s}} \in S} e^{\mathrm{i} k_{\mathrm{s}} \hat{\boldsymbol{s}} \cdot\left(\boldsymbol{y}-\boldsymbol{y}_{0}\right)} \mathcal{T}_{i, L}^{k, \mathrm{~S}}\left(\hat{\boldsymbol{s}} ; \boldsymbol{r}_{0}\right) e^{-\mathrm{i} k_{\mathrm{s}} \hat{\boldsymbol{s}} \cdot\left(\boldsymbol{x}-\boldsymbol{x}_{0}\right)} \mathrm{d} \hat{\boldsymbol{s}},
\end{aligned}
$$

with the elastodynamic transfer functions given in terms of the acoustic transfer function $\mathcal{G}_{L}$ by

$$
\begin{aligned}
& \mathcal{U}_{i, L}^{k, \mathrm{P}}\left(\hat{\boldsymbol{s}} ; \boldsymbol{r}_{0}\right)=\frac{\gamma^{2}}{\mu} \hat{s}_{i} \hat{s}_{k} \mathcal{G}_{L}\left(\hat{\boldsymbol{s}} ; \boldsymbol{r}_{0} ; k_{\mathrm{P}}\right), \\
& \mathcal{T}_{i, L}^{k, \mathrm{P}}\left(\hat{\boldsymbol{s}} ; \boldsymbol{r}_{0}\right)=\frac{\mathrm{i} k_{\mathrm{S}} \gamma^{3}}{\mu} C_{i j h \ell} \hat{s}_{\ell} \hat{s}_{h} \hat{s}_{k} \mathcal{G}_{L}\left(\hat{\boldsymbol{s}} ; \boldsymbol{r}_{0} ; k_{\mathrm{P}}\right) n_{j}(\boldsymbol{y}), \\
& \mathcal{U}_{i, L}^{k, \mathrm{~S}}\left(\hat{\boldsymbol{s}} ; \boldsymbol{r}_{0}\right)=\frac{1}{\mu}\left(\delta_{i k}-\hat{s}_{k} \hat{s}_{i}\right) \mathcal{G}_{L}\left(\hat{\boldsymbol{s}} ; \boldsymbol{r}_{0} ; k_{\mathrm{S}}\right), \\
& \mathcal{T}_{i, L}^{k, \mathrm{~S}}\left(\hat{\boldsymbol{s}} ; \boldsymbol{r}_{0}\right)=\frac{\mathrm{i} k_{\mathrm{S}}}{\mu}\left(\delta_{h k}-\hat{s}_{k} \hat{s}_{h}\right) C_{i j h \ell} \hat{s}_{\ell} \mathcal{G}_{L}\left(\hat{\boldsymbol{s}} ; \boldsymbol{r}_{0} ; k_{\mathrm{S}}\right) n_{j}(\boldsymbol{y}) .
\end{aligned}
$$

In practice, the limiting process $L \rightarrow+\infty$ in (8) or (10), (11) cannot be performed exactly and is replaced with an evaluation for a suitably chosen finite value of $L$, empirically established from numerical experiments. One such formula, known from previous studies on FMMs for Maxwell equations [7], reads:

$$
L(d)=\sqrt{3} k d+C_{\epsilon} \log _{10}(\sqrt{3} k d+\pi),
$$


where $d$ denotes the linear cell size. In this work, distinct truncation levels $L_{\mathrm{P}}$ and $L_{\mathrm{S}}$ are defined according to (13) with $k=k_{\mathrm{P}}$ and $k=k_{\mathrm{S}}$, respectively.

\subsection{Single-region FM-BEM}

To have maximal efficiency, FM-BEM algorithms must confine non-FM calculations to the smallest possible portion of the boundary while clustering whenever possible the computation of influence terms into the largest possible non-adjacent groups. This is achieved by the multi-level FMM [7, 16, 21], based on using large cells and hierarchically subdividing each cell into $2 \times 2 \times 2=8$ children cubic cells. This cell-subdivision approach is systematized by means of an oct-tree structure of cells. The level $\ell=0$, composed of only one cubic cell containing the whole surface $\partial \Omega$, is the tree root. The level- 0 cell is divided into $2 \times 2 \times 2=8$ children cubic cells, which constitute the level $\ell=1$. All level- 1 cells being adjacent, the FMM cannot be applied to them. The level $\ell=2$ is then defined by dividing each level- 1 cell into 8 children cells, and so contains 64 cells. The subdivision process is further repeated until the finest level $\ell=\bar{\ell}$, implicitly defined by a preset subdivision-stopping criterion, is reached. Level $-\bar{\ell}$ cells are usually termed leaf cells. The FMM is applied from level $\ell=2$ to level $\ell=\bar{\ell}$, i.e. features $\bar{\ell}-1$ "active" levels.

The multi-level approach basically consists in first applying the FMM to all influence computations between disjoint level-2 cells (so as to use the largest clusters whenever possible), and then recursively tracing the tree downwards, applying the FMM to all interaction between disjoint level- $\ell$ cells that are children of adjacent level-( $\ell-1)$ cells (Fig. 2). Finally, interactions between adjacent leaf cells are treated using traditional (i.e. non FM-based) BE techniques. This approach thus minimizes the overall proportion of influence computations requiring the traditional treatment.

The computation of the discretized linear operator (2), i.e. of the matrix-vector product $[K]\{u\}$, by the multi-level elastodynamic FMM hence consists of the following main steps:

1. Initialization: compute multipole moments for all lowest-level cells $\mathcal{C}_{y}=\mathcal{C}_{y}^{\bar{\ell}}$ :

$$
\begin{aligned}
& \mathcal{R}_{k}^{\mathrm{S}, u}\left(\hat{\boldsymbol{s}} ; \mathcal{C}_{y}^{(\bar{\ell})}\right)=-\mathrm{i} k_{\mathrm{S}} A_{i j k}^{\mathrm{S}, u} \int_{\partial \Omega \cap \mathcal{C}_{y}^{(\bar{\ell})}} u_{i}(\boldsymbol{y}) n_{j}(\boldsymbol{y}) e^{\mathrm{i} k_{\mathrm{S}} \hat{\boldsymbol{s}} \cdot\left(\boldsymbol{y}-\boldsymbol{y}_{0}^{(\bar{\ell})}\right)} d S_{y} \\
& \mathcal{R}^{\mathrm{P}, u}\left(\hat{\boldsymbol{s}} ; \mathcal{C}_{y}^{(\bar{\ell})}\right)=-2 \mathrm{i} k_{\mathrm{S}} \gamma^{3} A_{i j}^{\mathrm{P}, u} \int_{\partial \Omega \cap \mathcal{C}_{y}^{(\bar{\ell})}} u_{i}(\boldsymbol{y}) n_{j}(\boldsymbol{y}) e^{\mathrm{i} k_{\mathrm{P}} \hat{\boldsymbol{s}} \cdot\left(\boldsymbol{y}-\boldsymbol{y}_{0}^{(\bar{\ell})}\right)} d S_{y} \\
& \mathcal{R}_{k}^{\mathrm{S}, t}\left(\hat{\boldsymbol{s}} ; \mathcal{C}_{y}^{(\bar{\ell})}\right)=\frac{1}{\mu} A_{k i}^{\mathrm{S}, t} \int_{\partial \Omega \cap \mathcal{C}_{y}^{(\bar{\ell})}} t_{i}(\boldsymbol{y}) e^{\mathrm{i} k_{\mathrm{s}} \hat{s} \cdot\left(\boldsymbol{y}-\boldsymbol{y}_{0}^{(\bar{\ell})}\right)} d S_{y} \\
& \mathcal{R}^{\mathrm{P}, t}\left(\hat{\boldsymbol{s}} ; \mathcal{C}_{y}^{(\bar{\ell})}\right)=\frac{\gamma^{2}}{\mu} \int_{\partial \Omega \cap \mathcal{C}_{y}^{(\bar{\ell})}} \hat{s}_{a} t_{a}(\boldsymbol{y}) e^{\mathrm{i} k_{\mathrm{P}} \hat{\boldsymbol{s}} \cdot\left(\boldsymbol{y}-\boldsymbol{y}_{0}^{(\bar{\ell})}\right)} d S_{y}
\end{aligned}
$$




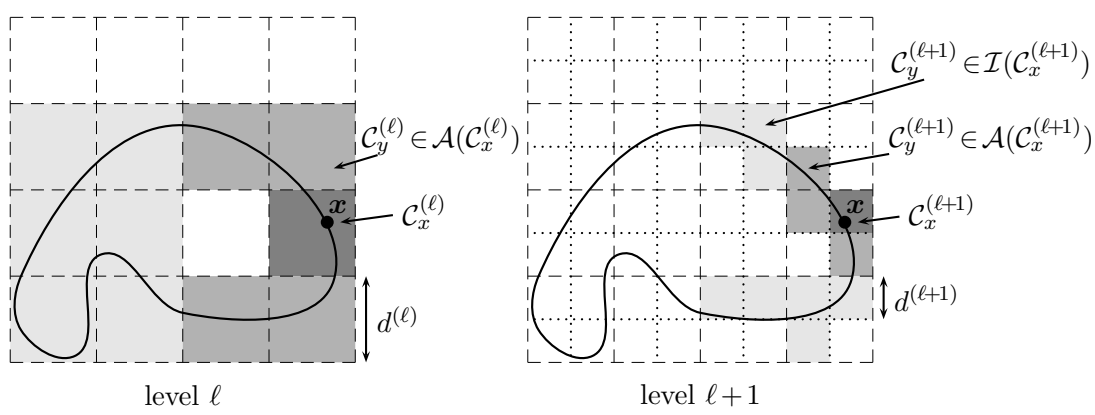

Fig. 2 Multi-level fast multipole algorithm. Only multipole moments from non-adjacent (lightgrey) cells $\mathcal{C}_{y}^{(\ell)} \notin \mathcal{A}\left(\mathcal{C}_{x}^{(\ell)}\right)$ may provide (through transfer) FM-computed contributions to $(\mathcal{K} u)^{\mathrm{FM}}(\boldsymbol{x})$ at collocation points $\boldsymbol{x}$ lying in cell $\mathcal{C}_{x}^{(\ell)}$. Upon cell subdivision (right), new FMcomputed contributions to collocation points in cell $\mathcal{C}_{x}^{(\ell+1)}$ originate from cells $\mathcal{C}_{y}^{(\ell+1)}$ in the interaction list $\mathcal{I}\left(\mathcal{C}_{x}^{(\ell+1)}\right)$ of $\mathcal{C}_{x}^{(\ell+1)}$, while the adjacent region $\mathcal{A}\left(\mathcal{C}_{x}^{(\ell+1)}\right)$ reduces in size.

having set $A_{i j k}^{\mathrm{S}, u}=\delta_{i k} \hat{s}_{j}+\delta_{j k} \hat{s}_{i}-2 \hat{s}_{i} \hat{s}_{j} \hat{s}_{k}, A_{i j}^{\mathrm{P}, u}=\nu(1-2 \nu)^{-1} \delta_{i j}+\hat{s}_{i} \hat{s}_{j}$ and $A_{k i}^{\mathrm{S}, t}=\delta_{k i}-\hat{s}_{k} \hat{s}_{i}$.

2. Upward pass: recursively aggregate multipole moments by moving upward in the tree until level 2 is reached. Denoting by $\mathcal{S}(\mathcal{C})$ the set of children of a given cell $\mathcal{C}$, the transition from a level- $(\ell+1)$ cell to its parent level- $\ell$ cell is based on identities

$$
\begin{aligned}
& \mathcal{R}_{k}^{\mathrm{S}, u}\left(\hat{\boldsymbol{s}} ; \mathcal{C}_{y}^{(\ell)}\right)=\sum_{\mathcal{C}_{y}^{(\ell+1)} \in \mathcal{S}\left(\mathcal{C}_{y}^{(\ell)}\right)} \exp \left[-\mathrm{i} k_{\mathrm{S}} \hat{\boldsymbol{s}} \cdot \Delta \boldsymbol{y}_{0}^{(\ell)}\right] \mathcal{R}_{k}^{\mathrm{S}, u}\left(\hat{\boldsymbol{s}} ; \mathcal{C}_{y}^{(\ell+1)}\right) \\
& \mathcal{R}^{\mathrm{P}, u}\left(\hat{\boldsymbol{s}} ; \mathcal{C}_{y}^{(\ell)}\right)=\sum_{\mathcal{C}_{y}^{(\ell+1)} \in \mathcal{S}\left(\mathcal{C}_{y}^{(\ell)}\right)} \exp \left[-\mathrm{i} k_{\mathrm{P}} \hat{\boldsymbol{s}} \cdot \Delta \boldsymbol{y}_{0}^{(\ell)}\right] \mathcal{R}^{\mathrm{P}, u}\left(\hat{\boldsymbol{s}} ; \mathcal{C}_{y}^{(\ell+1)}\right) .
\end{aligned}
$$

(with $\left.\Delta \boldsymbol{y}_{0}^{(\ell)}=\boldsymbol{y}_{0}^{(\ell+1)}-\boldsymbol{y}_{0}^{(\ell)}\right)$. A crucial feature of the elastodynamic multi-level FMM is that the number and location of the quadrature points on $S$ are leveldependent, a consequence of the previously-mentioned dependence (13) of $L$ on the cell size. Hence, application of identities $(15 \mathrm{a}, \mathrm{b})$ requires an extrapolation procedure [4,7] furnishing the values of $\mathcal{R}_{k}^{\mathrm{S}, u}$ and $\mathcal{R}^{\mathrm{P}, u}$ at the level- $\ell$ quadrature points from those at the level- $(\ell+1)$ quadrature points.

3. Transfer: initialize local expansions for each level- $\ell$ cell $\mathcal{C}_{x}^{(\ell)}$ and at each level $2 \leq \ell \leq \bar{\ell}$ using

$$
\begin{aligned}
& \mathcal{L}_{k}^{\mathrm{S}, u}\left(\hat{\boldsymbol{s}}^{(\ell)} ; \mathcal{C}_{x}^{(\ell)}\right)=\sum_{\mathcal{C}_{y}^{(\ell)} \in \mathcal{I}\left(\mathcal{C}_{x}^{(\ell)}\right)} \mathcal{G}_{L}\left(\hat{\boldsymbol{s}}^{(\ell)} ; \boldsymbol{r}_{0} ; k_{\mathrm{S}}\right) \mathcal{R}_{k}^{\mathrm{S}, u}\left(\hat{\boldsymbol{s}}^{(\ell)} ; \mathcal{C}_{y}^{(\ell)}\right) \\
& \mathcal{L}^{\mathrm{P}, u}\left(\hat{\boldsymbol{s}}^{(\ell)} ; \mathcal{C}_{x}^{(\ell)}\right)=\sum_{\mathcal{C}_{y}^{(\ell)} \in \mathcal{I}\left(\mathcal{C}_{x}^{(\ell)}\right)} \mathcal{G}_{L}\left(\hat{\boldsymbol{s}}^{(\ell)} ; \boldsymbol{r}_{0} ; k_{\mathrm{P}}\right) \mathcal{R}^{\mathrm{P}, u}\left(\hat{\boldsymbol{s}}^{(\ell)} ; \mathcal{C}_{y}^{(\ell)}\right)
\end{aligned}
$$


where $\mathcal{I}(\mathcal{C})$, the interaction list of a given cell $\mathcal{C}$ (Fig. 2), is the set of same-level cells which are not adjacent to $\mathcal{C}$ while having a parent cell adjacent to that of $\mathcal{C}$. For a level-2 cell, $\mathcal{I}\left(\mathcal{C}^{2}\right)$ collects all level-2 cells not adjacent to $\mathcal{C}^{2}$.

4. Downward pass: for all levels $3 \leq \ell \leq \bar{\ell}$, the local expansion for each level- $\ell$ cell $\mathcal{C}_{x}^{(\ell)}$ is updated with the contribution from the parent level- $(\ell-1)$ cell, by means of the identity

$$
\begin{aligned}
& \mathcal{L}_{k}^{\mathrm{S}, u}\left(\hat{\boldsymbol{s}} ; \mathcal{C}_{x}^{(\ell)}\right)=\mathcal{L}_{k}^{\mathrm{S}, u}\left(\hat{\boldsymbol{s}} ; \mathcal{C}_{x}^{(\ell)}\right)+\exp \left[-\mathrm{i} k_{\mathrm{S}}\left(\hat{\boldsymbol{s}} . \Delta \boldsymbol{x}_{0}^{(\ell)}\right)\right] \mathcal{L}_{k}^{\mathrm{S}, u}\left(\hat{\boldsymbol{s}} ; \mathcal{C}_{x}^{(\ell-1)}\right) \\
& \mathcal{L}^{\mathrm{P}, u}\left(\hat{\boldsymbol{s}} ; \mathcal{C}_{x}^{(\ell)}\right)=\mathcal{L}^{\mathrm{P}, u}\left(\hat{\boldsymbol{s}} ; \mathcal{C}_{x}^{(\ell)}\right)+\exp \left[-\mathrm{i} k_{\mathrm{P}}\left(\hat{\boldsymbol{s}} . \Delta \boldsymbol{x}_{0}^{(\ell)}\right)\right] \mathcal{L}^{\mathrm{P}, u}\left(\hat{\boldsymbol{s}} ; \mathcal{C}_{x}^{(\ell-1)}\right)
\end{aligned}
$$

(with $\Delta \boldsymbol{x}_{0}^{(\ell)}=\boldsymbol{x}_{0}^{(\ell-1)}-\boldsymbol{x}_{0}^{(\ell)}$ ). Similarly to step 2, application of identity (17a,b) requires an inverse extrapolation procedure [4,7] furnishing the values of $\mathcal{L}_{k}^{\mathrm{S}, u}$ and $\mathcal{L}^{\mathrm{P}, u}$ at the level- $\ell$ quadrature points from those at the level- $(\ell-1)$ quadrature points.

5. When the leaf level $\ell=\bar{\ell}$ is reached, all local expansions have been computed. The far contribution $(\mathcal{K} u)^{\mathrm{FM}}(\boldsymbol{x})$ is evaluated using

$$
\begin{aligned}
(\mathcal{K} u)_{k}^{\mathrm{FM}}(\boldsymbol{x}) \approx \sum_{q=1}^{Q} w_{q}^{(\bar{\ell})}\left[e^{-\mathrm{i} k_{\mathrm{s}} \hat{\boldsymbol{s}}_{q}^{(\bar{\ell})} \cdot\left(\boldsymbol{x}-\boldsymbol{x}_{0}^{(\bar{\ell})}\right)} \mathcal{L}_{k}^{\mathrm{S}, u}\left(\hat{\boldsymbol{s}}_{q} ; \mathcal{C}_{x}^{(\bar{\ell})}\right)\right. & \\
& \left.+e^{-\mathrm{i} k_{\mathrm{p}} \hat{\boldsymbol{s}}_{q} \cdot\left(\boldsymbol{x}-\boldsymbol{x}_{0}^{(\bar{\ell})}\right)}\left(\hat{\boldsymbol{s}}_{q}^{(\bar{\ell})}\right)_{k} \mathcal{L}^{\mathrm{P}, u}\left(\hat{\boldsymbol{s}}_{q} ; \mathcal{C}_{x}^{(\bar{\ell})}\right)\right],
\end{aligned}
$$

and the near contribution is evaluated for all level- $\bar{\ell}$ (leaf) cells $\mathcal{C}_{x}$ according to

$$
\begin{aligned}
(\mathcal{K} u)_{k}^{\text {near }}(\boldsymbol{x})=c_{i k}(\boldsymbol{x}) & u_{i}(\boldsymbol{x}) \\
+ & \sum_{\mathcal{C}_{y}^{(\bar{\ell})} \in \mathcal{A}\left(\mathcal{C}_{x}^{(\bar{\ell})}\right)}(\text { P.V. }) \int_{\partial \Omega \cap \mathcal{C}_{y}^{(\bar{\ell})}} u_{i}(\boldsymbol{y}) T_{i}^{k}(\boldsymbol{x}, \boldsymbol{y}) d S_{y}
\end{aligned}
$$

The computation of the right-hand side (3) follows the same steps, with the multipole moments $\mathcal{R}_{k}^{\mathrm{S}, u}, \mathcal{R}^{\mathrm{P}, u}$ and local expansions $\mathcal{L}_{k}^{\mathrm{S}, u}, \mathcal{L}^{\mathrm{P}, u}$ replaced with their counterparts $\mathcal{R}_{k}^{\mathrm{S}, t}, \mathcal{R}^{\mathrm{P}, t}$ and $\mathcal{L}_{k}^{\mathrm{S}, t}, \mathcal{L}^{\mathrm{P}, t}$. The above steps are found [4] to have a complexity of at most $O(N \log N)$, with the exception of the direct and inverse extrapolations in steps 2 and 4, whose complexity is $O\left(N^{3 / 2}\right)$.

The near-field contribution (19) involves (i) CPV-singular, (ii) weakly-singular and (iii) non-singular element integrals. CPV-singular integrals are split into (singular) integrals involving the static Kelvin traction kernel and (nonsingular) complementary integrals. The former are then evaluated analytically, taking advantage of the fact that three-noded triangular elements, which have constant unit normal and Jacobian, are used. Weakly-singular integrals (which feature the kernel $U_{i}^{k}(\boldsymbol{x}, \boldsymbol{y})$ ) and non-singular integrals are computed using numerical Gaussian quadrature (the weak singularity being first cancelled by means of a suitable change of coordinates). Finally, when $\partial \Omega$ presents an edge or corner at $\boldsymbol{x}$, the free-term $c_{i j}(\boldsymbol{x})$ is evaluated using the method of [14]. 
A detailed account of the implementation of this single-region elastodynamic FMM is described in [4], wherein analytical and numerical verifications of the algorithmic complexity of single-level and multi-level versions are also addressed.

\subsection{Multi-region FM-BEM}

The above FM-BEM formulation can be naturally extended to multi-domain configurations. Such problems involve displacement and traction degrees of freedom associated with interfacial displacements $\boldsymbol{u}^{i j}$ or $\boldsymbol{t}^{i j}$ on the common interface $\Gamma_{i j}$ separating subregions $\Omega_{i}$ and $\Omega_{j}$ (with $\boldsymbol{t}^{i j}$ conventionally defined in terms of the unit normal $\boldsymbol{n}^{i j}$ directed from $\Omega_{i}$ to $\Omega_{j}$ ). Invoking the perfect bonding conditions

$$
\boldsymbol{u}^{i j}=\boldsymbol{u}^{j i}, \quad \boldsymbol{t}^{i j}=-\boldsymbol{t}^{j i}
$$

the interfacial quantities $\boldsymbol{u}^{i j}, \boldsymbol{t}^{i j}$ with $i<j$ are retained as independent unknowns. For each subdomain $\Omega_{i}$, the governing integral equation (1) is discretized by collocation at the displacement nodes and the element centres. This defines for each subregion a rectangular, overdetermined system of BEM equations. The FM-BEM then evaluates for each $\Omega_{i}$ the corresponding matrix-vector product. To define a square global system of equations, equations resulting from collocation at interface element nodes or centres arising from both adjacent subdomains are linearly combined, an operation that is performed externally on the matrix-vector products generated by the FM-BEM. A detailed presentation of this BEM-BEM coupling approach, including numerical experiments allowing to select suitable values of the weighting coefficients used in the foregoing equation combinations, is given in [5].

\section{Numerical examples}

Following are a few sample numerical results obtained on test problems using the present elastodynamic single-region or multi-region FM-BEMs. Other examples are presented in [4] and [5].

\subsection{Scattering of $S V$ waves by a semi-spherical canyon}

This example is concerned with the perturbation by a semi-spherical canyon of radius $a$ of an oblique incident plane SV-wave of unit amplitude traveling in a elastic half space (Fig. 3) characterized by $\nu=1 / 3$. This problem has been previously studied in [10] by means of a wave function expansion and, for low frequencies, in [17] using a standard BEM. Results obtained for a low frequency such that 


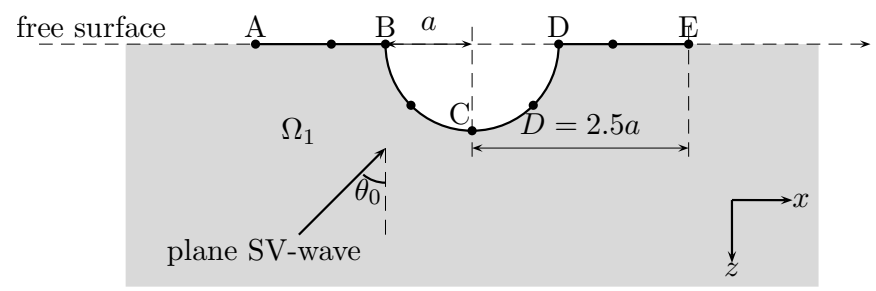

Fig. 3 Scattering of SV waves by a semi-spherical canyon: geometry and notation

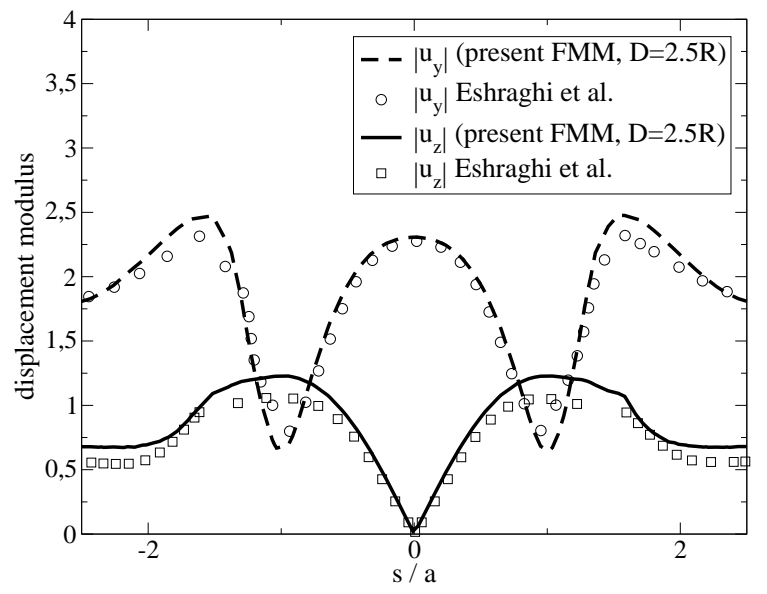

Fig. 4 Scattering of SV waves by a semi-spherical canyon: horizontal and vertical computed displacement on line ABCDE (with points A, B, C, D, E defined on Fig. 3) plotted against normalized arc-length coordinate $s / a$ along $\operatorname{ABCDE}\left(\theta=0, k_{\mathrm{S}} a=0.75 \pi\right)$

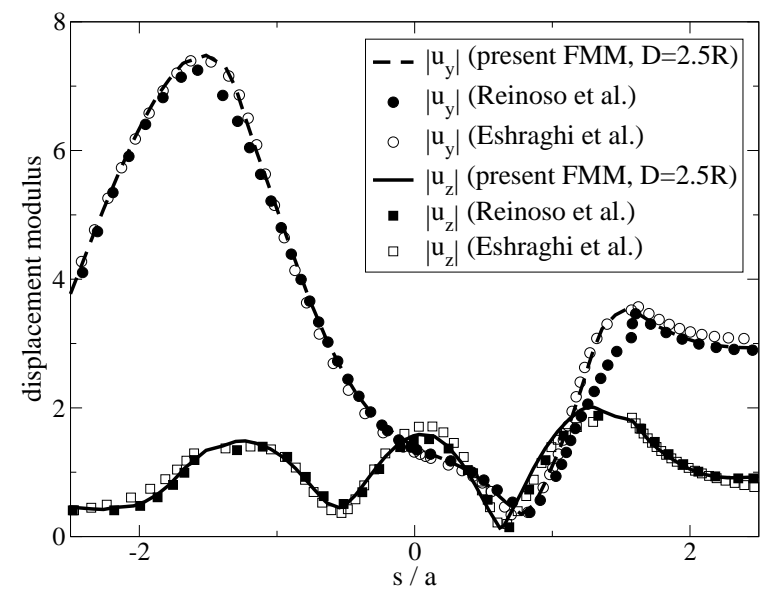

Fig. 5 Scattering of SV waves by a semi-spherical canyon: horizontal and vertical computed displacement on line ABCDE (with points A, B, C, D, E defined on Fig. 3) plotted against normalized arc-length coordinate $s / a$ along $\operatorname{ABCDE}\left(\theta=\pi / 6, k_{\mathrm{S}} a=0.75 \pi\right)$ 
$k_{\mathrm{S}} a=0.75 \pi$ are seen to agree well with published results from [10] $(\theta=0$, Fig. 4) and $[10,17](\theta=\pi / 6$, Fig. 5). The free surface is here meshed within a truncation radius $D=2.5 a$, a relatively small value chosen so as to reproduce the conditions used in [10,17], and the mesh features $N=7,602$ nodal unknowns overall. The computation required 8 (resp. 11) GMRES iterations for the case $\theta=0$ (resp. $\theta=\pi / 6$ ) and $1.5 \mathrm{~s}$ of CPU per iteration on a single-processor PC (RAM: 3GB, CPU frequency: $3.40 \mathrm{GHz}$ ), with the GMRES relative tolerance set to $10^{-3}$. The leaf level is $\bar{\ell}=3$, with the linear size $d^{\mathrm{min}}$ of leaf cells such that $k_{\mathrm{S}} d^{\min } \approx 1.45$.

\subsection{Test problem for the multi-region FMM}

The test problem of a spherical cavity subjected to an internal time-harmonic uniform pressure $P$, surrounded by two concentric spherical layers $\Omega_{1}, \Omega_{2}$ embedded in an unbounded elastic medium $\Omega_{3}$, is considered (Fig. 6). The radii of the cavity and two surrounding interfaces are $a_{1}, a_{2}, a_{3}$, respectively The mechanical properties of the respective media, in arbitrary units, are $\left(\rho_{1}, \mu_{1}, \nu_{1}\right)=(3,4,0.25)$, $\left(\rho_{2}, \mu_{2}, \nu_{2}\right)=(6,5,0.25),\left(\rho_{3}, \mu_{3}, \nu_{3}\right)=(2,1,1 / 3)$. A closed-form exact solution is available for this test problem. Results in terms of RMS errors between numerical solutions computed using the present multi-domain FM-BEM and their analytical counterparts, given in Table 1, show that the formulation achieves satisfactory accuracy (the GMRES relative tolerance being again set to $10^{-3}$ ).

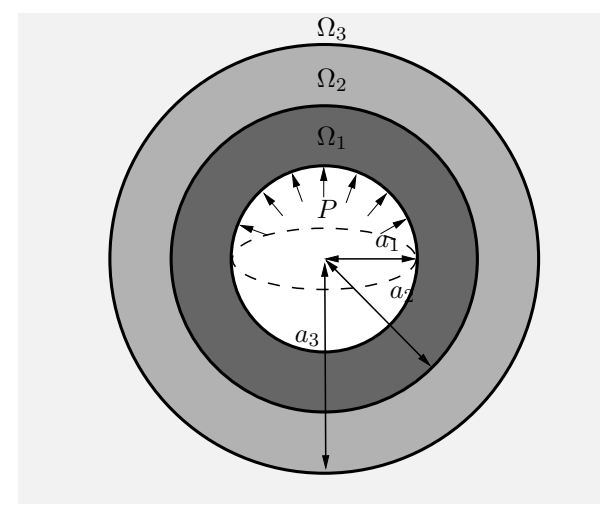

Fig. 6 Test problem: pressurized spherical cavity surrounded by concentric layers

Table 1 Pressurized spherical cavity surrounded by concentric layers: relative RMS errors

\begin{tabular}{cccccccccc}
\hline$N$ & $k_{\mathrm{S}}^{1} d^{\min }$ & $k_{S}^{1} a_{1}$ & $\bar{\ell}_{i}$ & $E\left(u^{1}\right)$ & $E\left(u^{12}\right)$ & $E\left(t^{12}\right)$ & $E\left(u^{23}\right)$ & $E\left(t^{23}\right)$ & Iters. \\
\hline 55,778 & 0.82 & 2.17 & $3 ; 3 ; 3$ & $3.010^{-2}$ & $1.410^{-2}$ & $2.210^{-2}$ & $1.310^{-2}$ & $2.810^{-2}$ & 59 \\
215,058 & 1.88 & 4.93 & $3 ; 3 ; 4$ & $1.010^{-2}$ & $1.310^{-2}$ & $1.010^{-2}$ & $1.410^{-2}$ & $1.410^{-2}$ & 43 \\
\hline
\end{tabular}




\subsection{Wave amplification in a semi-spherical basin}

This example is again concerned with the perturbation of an oblique incident plane SV-wave of unit amplitude traveling in a elastic half space, this time by a semispherical filled basin (Fig. 7). This configuration, has been studied in the frequency domain in [15] using a standard indirect BEM (using the half-space Green's functions). The mechanical parameters are defined through $\mu_{1}=c_{\mathrm{S} 1}=1, c_{\mathrm{P} 1}=2$, $\mu_{2}=1 / 6, c_{\mathrm{S} 2}=1 / 2$ and $c_{\mathrm{P} 2}=1$. Results obtained for an incidence angle $\theta=\pi / 6$ and a frequency such that $k_{\mathrm{S}} a=2 \pi_{1}$ are presented, in terms of the $x, y$ and $z$ components of the surface displacement, in Figures 8, 9 and 10. The free surface is here meshed within a truncation radius $D=5 a$, and the mesh features $N=143,451$ nodal unknowns (discretized versions of the displacement $\boldsymbol{u}^{1}$ on the truncated free surface and the interfacial displacements $\boldsymbol{u}^{12}$ and tractions $\boldsymbol{t}^{12}$ ). The computation required 484 GMRES iterations and 36s of CPU per iteration.

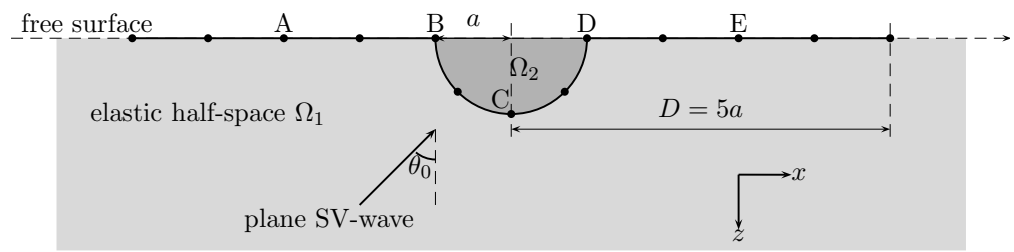

Fig. 7 Propagation of an oblique incident plane SV-wave in a semi-spherical basin: notation.

\section{Conclusions}

In this contribution, the Fast Multipole Method has been succesfully extended to $3 D$ single-region and multi-region elastodynamics in the frequency domain. Combined with the BEM formulation, it permits to reduce the computational burden, in both CPU time and memory requirements, for the analysis of elastic wave propagation (e. g. seismic), and allows to run BEM models of large size on an ordinary PC. Comparisons with analytical or previously published numerical results show the efficiency and accuracy of the present elastodynamic FM-BEM.

Applications of the present FM-BEM to realistic cases in seismology are under way. Moreover, a natural extension of this work consists in formulating multipole expansions of other fundamental solutions, with the half-space elastodynamic fundamental solution being currently investigated. Also, extending the formulation to complex wavenumbers will allow more realistic modelling where viscoelastic constitutive properties are assumed for the propagation medium. Finally, improving the efficiency of the elastodynamic FM-BEM also requires further research into refined 


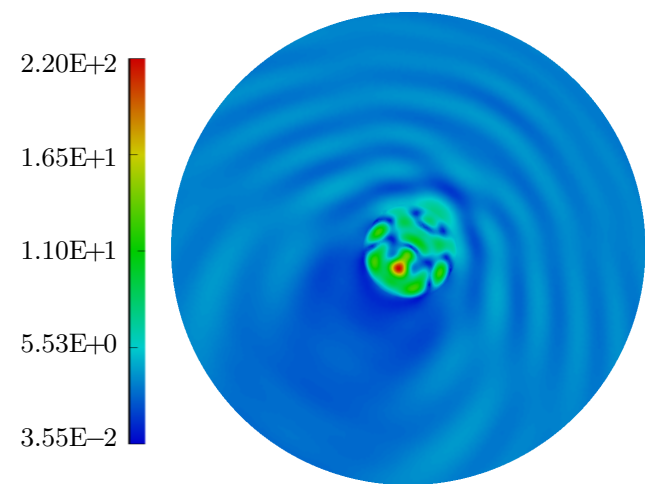

Fig. 8 Diffraction of an oblique incident SV plane wave by a semi-spherical basin: computed $x$ component of displacement on basin surface and meshed part of free surface (normalized frequency $\left.k_{\mathrm{S}} a=2 \pi\right)$

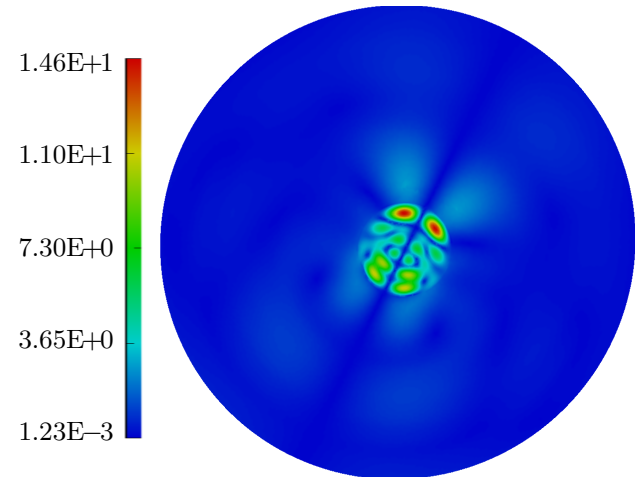

Fig. 9 Diffraction of an oblique incident SV plane wave by a semi-spherical basin: computed $y$ component of displacement on basin surface and meshed part of free surface (normalized frequency $\left.k_{\mathrm{S}} a=2 \pi\right)$

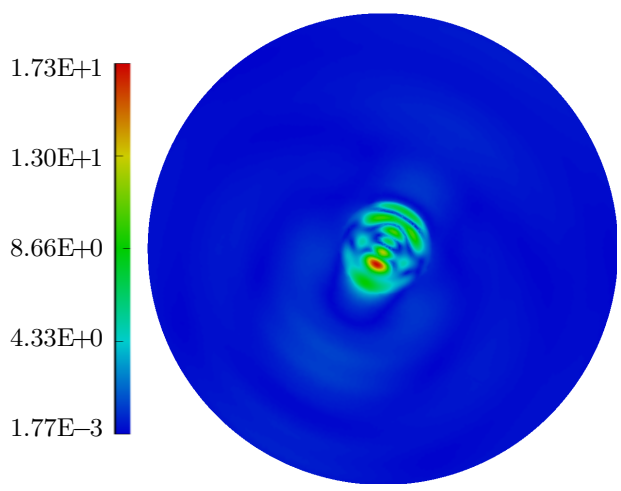

Fig. 10 Diffraction of an oblique incident SV plane wave by a semi-spherical basin: computed $z$ component of displacement on basin surface and meshed part of free surface (normalized frequency $\left.k_{\mathrm{S}} a=2 \pi\right)$ 
(direct/inverse) extrapolation techniques (for lowering the $O\left(N^{3 / 2}\right)$ of this step) and a well-chosen preconditioning strategy (for reducing the GMRES iteration count).

Acknowledgements This work is part of the project Quantitative Seismic Hazard Assessment (QSHA, http://qsha.unice.fr) funded by the French National Research Agency (ANR)

\section{References}

1. Beskos, D. Boundary element methods in dynamic analysis. Appl. Mech. Rev., 40:1-23 (1987).

2. Beskos, D. Boundary element methods in dynamic analysis, part. II. Appl. Mech. Rev., 50:149197 (1997).

3. Bonnet, M. Boundary Integral Equation Method for Solids and Fluids. Wiley (1999).

4. Chaillat, S., Bonnet, M., Semblat, J. F. A multi-level fast multipole BEM for 3-D elastodynamics in the frequency domain. Comp. Meth. Appl. Mech. Engng., in press (2008).

5. Chaillat, S., Bonnet, M., Semblat, J. F. A new fast multi-domain BEM to model seismic wave propagation and amplification in 3D geological structures. In preparation (2008).

6. Dangla, P., Semblat, J. F., Xiao, H., Delépine, N. A simple and efficient regularization method for 3D BEM: application to frequency-domain elastodynamics. Bull. Seism. Soc. Am., 95:1916-1927 (2005).

7. Darve, E. The Fast Multipole Method : Numerical Implementation. J. Comp. Phys., 160:195240 (2000).

8. Epton, M.A., Dembart, B. Multipole translation theory for the three-dimensional Laplace and Helmholtz equations. SIAM J. Sci. Comp., 16:865-897 (1995).

9. Eringen, A.C., Suhubi, E.S. Elastodynamics, vol. II-linear theory. Academic Press (1975).

10. Eshraghi, H., Dravinski, M. Scattering of plane harmonic SH, SV, P and Rayleigh waves by non-axisymmetric three-dimensional canyons: a wave function expansion approach. Earthquake Engng. and Struct. Dyn., 18:983-998 (1989).

11. Fujiwara, H. The fast multipole method for solving integral equations of three-dimensional topography and basin problems. Geophys. J. Int., 140:198-210 (2000).

12. Gumerov, N. A., Duraiswami, R. Fast multipole methods for the Helmholtz equation in three dimensions. Elsevier (2005).

13. Guzina, B. B., Pak, R. Y. S. On the Analysis of Wave Motions in a Multi-Layered Solid. Quart. J. Mech. Appl. Math., 54:13-37 (2001).

14. Mantic, V. A new formula for the C-matrix in the Somigliana identity. J. Elast., 33:191-201 (1993).

15. Mossessian, T.K., Dravinski, M. Amplification of elastic waves by a three dimensional valley. Part 1: steady state response. Earthquake Engng. Struct. Dyn., 19:667-680 (1990).

16. Nishimura, N. Fast multipole accelerated boundary integral equation methods. Appl. Mech. Rev., 55(4) (2002)

17. Reinoso, E., Wrobel, L. C., Power, H. Three-dimensional scattering of seismic waves from topographical structures. Soil. Dyn. Earthquake Engng., 16:41-61 (1997).

18. Rokhlin, V. Diagonal forms of translation operators for the Helmholtz equation in three dimensions. Appl. Comp. Harmonic Anal., 1:82-93 (1993).

19. Saad, Y., Schultz, M.H. GMRES: a generalized minimal residual algorithm for solving nonsymmetric linear systems. SIAM J. Sci. Stat. Comput., 7:856-869 (1986).

20. Sánchez-Sesma, F. J. Diffraction of elastic waves by 3D surface irregularities. Bull. Seism. Soc. Am., 73:1621-1636 (1983).

21. Sylvand, G. La méthode multipôle rapide en éléctromagnétisme : performances, parallélisation, applications. Ph.D. thesis, ENPC, Paris, France (2002). 